\title{
Managing variation to produce quality in farm products
}

\author{
A.B. PLEASANTS ${ }^{1}$, R. FORGIE ${ }^{2}$ and G.W. SHEATH ${ }^{1}$ \\ ${ }^{1}$ Whatawhata Research Centre, AgResearch, Private Bag 3089, Hamilton, New Zealand \\ ${ }^{2}$ AFFCO New Zealand Ltd., PO Box 90, Auckland, New Zealand
}

\begin{abstract}
The fundamental influence of variation on the production of quality products is reviewed. The partitioning of this variation into that which is controllable on the farm and that which is uncontrollable on the farm is discussed. The effectiveness of an on-farm quality assurance programme depends on the level of variation in a trait than can be controlled by farm management practices. A simple graphical tool that can be used on farm and is suitable for monitoring performance through time is the cusum control chart. This chart plots the cumulative sum of deviations from target performance. It is designed to identify the occurrence of problems in production on farm for which effective management action may be taken. The operation of a cusum chart for monitoring a quality assurance programme on farm is illustrated, using as an example the control of ultimate muscle $\mathrm{pH}$ in cattle. The cusum chart in this example is designed and operated using measurements supplied by a meat processor which were made on the muscle following slaughter.
\end{abstract}

Keywords: controllable variation, cusum chart, farm monitoring, quality assurance, uncontrollable variation

\section{What does quality mean?}

Unfortunately quality is an ambiguous term in everyday use. It can be taken to mean something vaguely "fine", and therefore determined by the eye of the beholder. But this is not what quality means in modern production processes. In this context a quality product is a product that is "fit for use". The concept "fit for use" focuses attention on the customer's expectation of the product. Quality products must satisfy customer needs.

Customer needs can be defined through surveys and other methods employed by market researchers. These needs can be summarised into a protocol called the product specifications for the market of interest. For example, a restaurant chain may have needs summarised in specifications that steaks be a defined size, of a defined tenderness, and delivered fresh within a defined time. Steaks which meet these requirements are considered quality steaks by the restaurant chain.

The issue in producing quality products is recognition that all products are never the same, especially biological products. Variation exists, and the basic problem of quality assurance is how to manage this variation within acceptable tolerances. This paper describes objective methods of measuring variation and introduces a simple graphical tool, the cusum chart, which can be used as an aid to managing variation in carcass traits on a farm.

\section{The association of product quality with product variation}

The role of variation in product quality, or in deciding the "fitness of use" of the product, can be best illustrated through a situation which will be familiar to farmers. Consider a decision to buy $10-\mathrm{mm}$ diameter bolts to build a gate. The use of the bolt in this situation is to hold the planks of the gate together, and the specifications for this task call for the bolts to have a diameter of $10 \mathrm{~mm}$. Not all bolts in the lot will be exactly $10 \mathrm{~mm}$. If variation in the lot of bolts ranges over $1 / 10 \mathrm{~mm}$, then there will be no discernible effect on the functionality of the bolt - i.e., the ability of the bolts to hold the gate together tightly. But if the variation in the bolts ranges around a $10 \mathrm{~mm}$ average by $\pm 2.5 \mathrm{~mm}$ a high proportion of the bolts would be unusable. Some would be too big, others too small. Bolts that were too small could not hold the gate planks tightly and the gate would not hold together. Bolts that were too big would not fit the holes drilled for them. If the bolts were bought for the one job of building the gate, a lot of bolts would be thrown away. This would be likely to make the cost per usable bolt of building the gate unacceptably high. A better deal would be to pay more for bolts of less variation in the knowledge that though such bolts were more expensive the extra cost would be recouped by having less wastage. Alternatively, if it were known that variation in the bolt size was high a customer would wish to discount the value of such bolts to compensate for the expected wastage.

A similar situation occurs for the customers of our farm products. If a restaurant chain ends up with a range of steak sizes some which are too big and others 
too small for the dishes it wishes to serve, then a proportion will be unusable, even though the average steak size might be exactly to the specification. This increases costs to the restaurant chain because they will need to get rid of unusable steaks, and introduces uncertainty about how much steak will need to be ordered.

As these examples show, the amount of variation in a product in relation to the intended use of the product is an important issue in customer satisfaction. Reducing variation increases the scope of markets for a product, and brings better returns to the producer. The relationship of product variation to quality is discussed by Murdoch (1979).

\section{Describing and partitioning variation in a trait}

Variation in a trait can be visualised with a graph of frequencies as illustrated in Figure 1. This shows the frequency distribution for the ultimate muscle $\mathrm{pH}$ of steers slaughtered at Manawatu Beef Packers in 1993/ 1994. The more spread out the graph, the greater the variation in the trait.

Figure 1 Frequency distribution of ultimate muscle $\mathrm{pH}$ for 15625 steer carcasses of various breeds measured post slaughter between Februray 1993 and January 1994 at Manawatu Beef Packers (AFFCo).

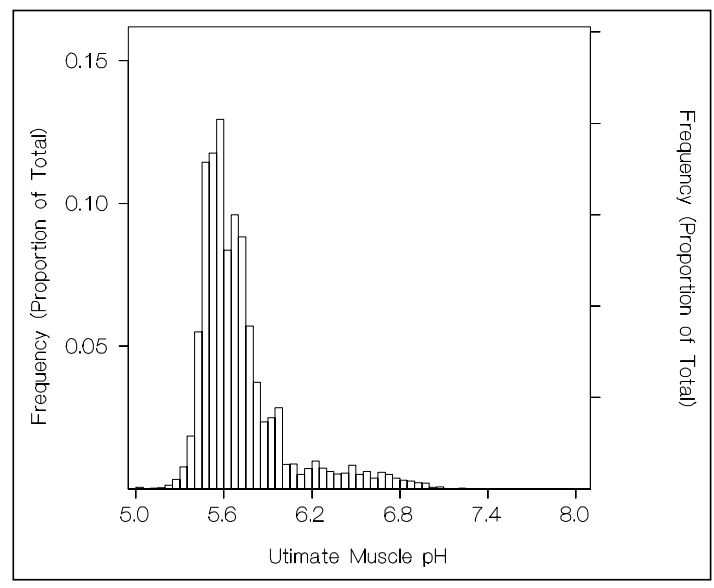

Variation in a trait like carcass weight will be due to the sum of variation from various environmental and growth effects that influence carcass weight. Such summation means that the sources of variation in a trait like carcass weight can be attributed to the action of different factors. The estimation of the effects of different factors on the variation of a trait is called partitioning the variation among the different sources.

Variation in a trait can be partitioned between the variation that can be controlled by a farmer through management practices (e.g., choosing animals of similar genetic merit), and variation which cannot be controlled in this way. In Figure 1 controllable variation due to breed, farmer, and time of the year have been removed through analysis. Thus the frequency distribution of Figure 1 measures the variation in ultimate muscle $\mathrm{pH}$ for which we do not know the reason. That is, it is the uncontrollable variation in ultimate muscle $\mathrm{pH}$ from the farm, since if the reason for the variation is not known no effective action can be taken by a farmer to reduce it.

Partitioning variation into economically controllable and uncontrollable variation is the first step in designing an on-farm quality assurance scheme. It is foolish to attempt to control the uncontrollable, or to waste effort which does not return a net benefit. From such an analysis the cost and effectiveness of a quality assurance scheme can be decided.

\section{Implementation of farm quality assurance}

An effective on-farm quality assurance scheme needs a decision rule to decide when action needs to be taken by the farmer to improve a trait associated with quality. Such a decision rule needs to acknowledge:

- The existence of uncontrollable variation

- The costs and benefits of taking action.

To illustrate the principles of on-farm quality assurance we consider the ultimate $\mathrm{pH}$ of the muscle of slaughtered cattle. Ultimate muscle $\mathrm{pH}$ has been associated with the tenderness and keeping qualities of the meat (Judd 1994; Purchas 1990). Muscle pH was measured with a $\mathrm{pH}$ meter in the meat processing plant after slaughter. However, some measurements were taken less than 20 hours after slaughter, and thus may not be the ultimate muscle $\mathrm{pH}$. Thus, the uncontrollable variation in this trait may be reduced by further standardisation.

Uncontrollable variation in a trait such as ultimate muscle $\mathrm{pH}$ means that there will almost always be some animals in a mob of cattle submitted for slaughter which will have unacceptable muscle $\mathrm{pH}$. It is axiomatic that any action taken to try to correct uncontrollable variation will be ineffective; therefore a reliable decision rule needs to take account of the level of uncontrollable variation in a trait. A simple graphical tool which can be used to do this is the cusum chart (Wetherill 1977). 


\section{The cusum control chart}

The cusum chart is a plot through time of the difference in a trait between the average of a sample of animals and the nominated target for the trait. This difference is standardised by dividing by the standard deviation of the average of the sample before plotting. This accounts for different samples that have more or less variation, and are therefore more or less reliable in their estimates. Each standardised deviation is added to a running sum and plotted on a graph.

If the overall average of production is at the target value it would be expected that there would be an approximately equal number of positive and negative deviations measured from target over time. The cusum plot would be expected to fluctuate about zero. However, if a change of management strategy or some other factor occurred that (say) increased the average of the trait of interest then there would be more positive deviations than negative deviations and the cusum plot would show an upward trend away from zero. A cusum chart is shown in Figure 2 for muscle $\mathrm{pH}$ of slaughtered steers using a target $\mathrm{pH}$ of 5.8 . Before sample number 21 the sample average deviations for muscle $\mathrm{pH}$ fluctuate around zero, indicating that the overall average of production is less than or equal to $\mathrm{pH} 5.8$ since only positive trends are plotted. At sample 21 the average muscle $\mathrm{pH}$ in the slaughtered animals increases to 6.2, and there is a rapid increase in the cusum plot. A decision line on the cusum chart is drawn at standardised cusum of 2. When the cusum plot crosses this line unacceptable variation in the trait is deemed to have occurred and management action is necessary.

Because of natural uncontrollable variation in a trait some trends in the cusum plot would occur by chance. The problem is to decide when an observed trend is due to a real effect that deserves attention from the manager, and when a trend may be attributed to natural variation. The design of the cusum chart, in particular the position to place the decision line, addresses this question.

The goal in designing a cusum chart is to have a decision support tool which, when a problem occurs, has a high probability of signalling a problem within a few successive plots on the cusum chart. On the other hand, when there is no problem, and cusum trends are due to uncontrollable variation, the cusum should make very few false signals. To achieve these aims a designer can manipulate the target value of the trait and one or more decision lines. The details in the design of cusum charts for meat traits associated with quality, including the placement of the decision lines, the identification of targets and the estimation of the onset of
Figure 2 Cusum chart for ultimate muscle $\mathrm{pH}$ with decision line set at two standard deviation units. The cusum is measured in standard deviation units from target value of $\mathrm{pH}$ 5.8. Mob number are consecutive groups of animals slaughtered by a farmer.

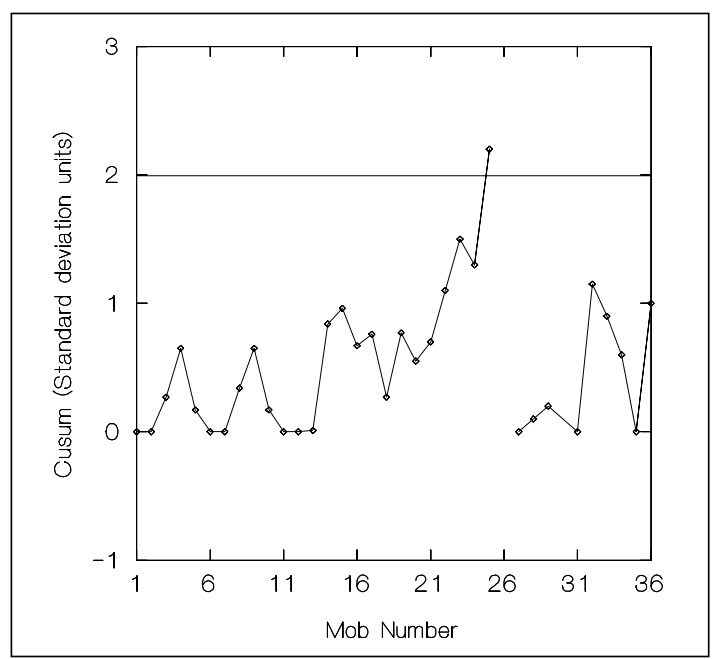

unacceptable production are given by Pleasants \& Sheath (unpublished).

The point at which a problem began can be estimated from the cusum plot. This assists the management in identifying and remedying the reason why production has become unacceptable. Figure 2 also shows the situation after some hypothetical corrective action has been taken. The cusum is restarted from zero and the succeeding plot shows action has been successful and that production is satisfactory.

A cusum chart will generally be specific to each farm. Different farms will have different aptitudes for collecting data and managing "out of control" events. Some farmers may have the scope to produce for markets which are particularly demanding in the performance of some traits. These farmers may need cusum charts for a wider range of traits, and with decision lines which are more stringent. Partnership between a farmer and a meat processor able to supply data and offer guidance in the design of cusum charts would assist such development.

Cusum charts can also be designed to detect changes in within-group variation over time. The operation of charts to detect changes in within-group variation is similar to the operation of charts to detect changes in trend. In this case estimates of variation are plotted. However, the design of cusum charts to monitor variation will be different to the design of cusum charts to monitor the average effect. 


\section{Conclusion}

Implementing quality assurance incurs cost to a farmer which will need to be recovered in the market-place. For example, improving the ultimate muscle $\mathrm{pH}$ may require investment in facilities designed to reduce stress on the animals sent for slaughter. Thus clear signals from the market about needs and returns are essential to development of on-farm quality assurance schemes. However, in the long term, investment in on-farm quality assurance may be the costs of staying in business. It can be expected that eventually customers will require quality accreditation before they will consider doing business. New Zealand agricultural industries need to prepare for this eventuality.

Quality assurance is a commitment by any manager to produce to specification within stated tolerances. It also implies a commitment to continual improvement of production systems, seeking methods to reduce tolerances still further using objective information gathered on the system. Information tools such as the cusum chart can assist this process. Better scientific understanding of the factors affecting the development of variation in important traits such as ultimate muscle pH will also be important. Such improved understanding can be easily designed into cusum charts.

Quality assurance is the new frontier for New Zealand agriculture. Successful implementation of quality assurance schemes on farm, and throughout the distribution network, will assist New Zealand agriculture to remain competitive in the future.

\section{ACKNOWLEDGMENTS}

D.G. McCall for assistance in the preparation of this manuscript.

\section{REFERENCES}

Murdoch, J. 1979. Control charts. MacMillan. London. Purchas, R.W. 1990. An assessment of the role of $\mathrm{pH}$ differences in determining the relative tenderness of meat from bulls and steers. Meat science 27: 129-140.

Wetherill, G.B. 1977. Sampling inspection and quality control. 2nd Edition. Chapman and Hall, London. 